\title{
Temporal grouping in auditory spatial serial memory
}

\author{
FABRICE B. R. PARMENTIER \\ University of Plymouth, Plymouth, England \\ MURRAY T. MAYBERY \\ University of Western Australia, Perth, Western Australia, Australia \\ and \\ DYLAN M. JONES \\ Cardiff University, Cardiff, Wales \\ and University of Western Australia, Perth, Western Australia, Australia
}

\begin{abstract}
Grouping effects in serial recall have been widely studied with verbal stimuli, but hardly ever with spatial stimuli and not at all with auditory spatial stimuli. In Experiment 1, we examined the influence of combined temporal and pitch grouping on recall of the locations from which bursts of white noise were presented. Similar to findings in verbal studies, effects of the grouping manipulation were found in performance accuracy, in the nature of order errors, and in the timing of responses. Experiment 2 was designed to distinguish the role played by pitch grouping from that played by temporal grouping, through independent manipulation of the presence of a shift in pitch and that of a temporal gap. The results showed that the temporal grouping manipulation determined performance and the pitch grouping manipulation did not. Similarities between our findings and those of verbal studies, and implications for the understanding of serial memory are discussed.
\end{abstract}

Grouping by proximity (e.g., by temporal proximity) constitutes a powerful method of encoding, maintaining, and retrieving information. Generally, temporal grouping is known to affect serial recall of lists of items in four ways. First, temporal grouping improves overall recall accuracy (see, e.g., Frankish, 1985, 1989). Second, it modifies the shape of the serial position curve in a characteristic fashion, creating within-group serial position effects, or scalloping (Hitch, Burgess, Towse, \& Culpin, 1996). Third, grouping generates specific classes of errors, although this is less well documented (see, e.g., Henson, 1998). For example, in a sequence of six items presented in groups (e.g., 123-456), migrations among items presented in Serial Positions 3 and 4 are less frequent than other adjacent migrations, whereas with ungrouped presentations (e.g., 123456) the reverse is found ( $\mathrm{Ng} \&$ Maybery, 2002). Finally, grouping has also been found to lead to changes in the timing of recall, indicating that participants group their responses according to the structure of

This research was supported by a large Australian Research Council (ARC) project grant to M.T.M., D. Morrison, and D.M.J., an ARC IREX travel grant to the same authors, and internal funding from the Department of Psychology at the University of Plymouth. Thanks are due Patricia Reuter-Lorenz, James Nairne, Gerry Tehan, and two anonymous reviewers for their useful suggestions during the review process. Thanks also to Greg Elford for helping with the collection of data. Correspondence can be addressed to F. Parmentier, Department of Psychology, University of Plymouth, Drake Circus, Plymouth PL4 8AA, England (e-mail: fparmentier@plymouth.ac.uk). the presented to-be-remembered list (Maybery, Parmentier, \& Jones, 2002). That is, in a list made of two groups separated by an extended time interval, a peak in response time (RT) was found at Serial Position 4 (the onset of the second group) relative to RTs at Serial Positions 3 and 5.

It is noteworthy that recent models of serial memory have been based mostly, if not entirely, on empirical studies focusing on verbal material (Anderson \& Matessa, 1997; Brown, Preece, \& Hulme, 2000; Page \& Norris, 1998), even though serial order is a critical dimension of many different types of activities other than language (see, e.g., Lashley, 1951). In the present study, we report the first empirical investigation of grouping of auditory spatial serial memory and seek to establish the degree of functional similarity in the action of grouping across spatial and verbal domains.

In order to promote grouping in Experiment 1, two types of cue were used in the expectation that they would act synergistically: temporal grouping (see, e.g., Hitch et al., 1996) and pitch grouping (Frankish, 1989). Participants were presented with sequences of six bursts of white noise mixed with a pure tone, played from different spatial locations around them. Their task was to report the order of the locations presented. Grouping consisted in inserting a longer gap than usual between Items 3 and 4 and changing the pitch of the stimuli in the two groups (low in one group and high in the other). If auditory spatial serial memory responds to manipulations in- 
tended to promote grouping as auditory verbal memory does, these manipulations should improve recall, produce within-group serial position effects, limit adjacent migrations across groups, and show a peak in RT for the transition between groups.

\section{EXPERIMENT 1}

\section{Method}

Participants. Twenty-four undergraduate psychology students at Cardiff University who reported normal hearing participated in exchange for course credit.

Apparatus and Materials. Eight loudspeakers were arranged around the participant at a radius of $1.2 \mathrm{~m}$, located at $-105^{\circ},-75^{\circ}$, $-45^{\circ},-15^{\circ},+15^{\circ},+45^{\circ},+75^{\circ}$, and $+105^{\circ}$ relative to the median plane, angles well within the localization abilities of humans for broadband signals (see, e.g., Oldfield \& Parker, 1984). The speakers were all at the same elevation, approximately at ear level for a seated participant. A computer screen was located in front of the participant, lower than the speakers' elevation to minimize sound diffusion. The loudspeakers were connected to a multitrack digital audiotape system via amplifiers. Sounds were played at an intensity of approximately $70 \mathrm{~dB}(\mathrm{~A})$ as measured by an audiometer. The stimuli were bursts of white noise mixed with either a $139-\mathrm{Hz}$ or a $196-\mathrm{Hz}$ sine-wave tone. All sounds were $600 \mathrm{msec}$ in duration (with $20 \mathrm{msec}$ of attack and $20 \mathrm{msec}$ of decay). Response options were displayed on the computer screen, and responses were collected via the computer mouse.

Design and Procedure. There were two sets of stimuli, each comprising 4 practice and 16 test sequences. Each sequence consisted of six speaker locations. The 16 test sequences for each set were constructed under the following constraints: (1) No speaker location was used more than once in any sequence, (2) adjacent speakers were not used for consecutive sequence items, (3) a speaker location was not presented in the same serial position in two successive sequences, and (4) across the 16 sequences, each speaker location was presented exactly twice in each serial position.

Two conditions, ungrouped and grouped, were presented in blocks, the order of which was counterbalanced across participants. The assignment of the two sets of memory items to the two conditions was balanced so that each set was presented in its grouped and ungrouped versions equally often.

In the ungrouped condition, the six stimuli were presented at a constant pitch $(139 \mathrm{~Hz}$ in half of the trials and $196 \mathrm{~Hz}$ in the remaining trials, randomly mixed). The two pitch values were taken from Frankish (1985, Experiment 4). The stimulus onset asynchrony (SOA) in the ungrouped condition was $900 \mathrm{msec}$ (stimulus "on" for $600 \mathrm{msec}$ and "off" for $300 \mathrm{msec}$ ). In the grouped condition, items were presented as two groups of three items by manipulating both their pitch $(139 \mathrm{~Hz}$ for one group and $196 \mathrm{~Hz}$ for the other, or vice versa, with each order used equally often across the 20 trials) and their timing (the within-group SOA was $650 \mathrm{msec}$, whereas the between-groups SOA was $1,900 \mathrm{msec}$ ). For both conditions, a time gap of $350 \mathrm{msec}$ separated offset of the last item from onset of the recall display, and the total duration of a sequence (onset of first item to onset of recall display) was 5,450 msec.

The participants sat in a darkened room. Each trial started with a warning signal (a high-pitched tone from the two front loudspeakers and the screen flashing blue) $3 \mathrm{sec}$ before the onset of item presentation. A cross was also presented at the center of the screen, which the participant fixated during the items' presentations. At recall, a diagram representing the loudspeakers (arc of circles) appeared, and an 18-sec response period was made available for the participants to click on the circles in the order in which the sounds were presented. When selected, each circle changed color and remained unavailable until the next trial. The participant could skip a particular item in the response sequence if he or she was unable to remember its location, by pressing a "skip" button displayed on the screen. Also displayed was a series of six squares arranged in a horizontal line to represent the responses to be collected. Squares filled in green, from left to right, as responses were produced.

\section{Results}

Three dependent variables were analyzed: accuracy, error rates, and RTs. A factor representing the order of presentation of the grouped and ungrouped conditions did not enter into any significant effects, and so it is not included in the analyses reported below. For clarity, only significant differences are presented.

Accuracy. Only items recalled in their correct serial positions were considered correct responses. The percentage of correct responses was analyzed in a 2 (condition: grouped vs. ungrouped) $\times 6$ (serial position) repeated measures ANOVA. The effect of condition was significant $\left[F(1,23)=8.57, M S_{\mathrm{e}}=277.85, p<.01\right]$, as were the effects of serial position $\left[F(5,115)=32.22, M S_{\mathrm{e}}=229.24\right.$, $p<.001]$ and interaction $\left[F(5,115)=4.19, M S_{\mathrm{e}}=134.68\right.$, $p<.005$; see Figure 1, top panel]. The analysis of the interaction revealed a significant advantage of grouping at Serial Position 3 only $[F(1,23)=21.35, p<.001]$.

Classification of errors. Different types of error, are possible in the task described above: omissions, intrusions, and order errors. Order errors constituted the large majority of the errors that we found, followed by intrusions and, finally, by omissions. As is reported in the Appendix, the grouping manipulation did not affect the frequency of omissions, intrusions, or order errors.

Although many order errors are possible, depending on the position of the item in the presented list and its erroneous position in the recalled list, a key prediction with regard to grouping lies with particular types of order errors representing migrations of items to a nearby serial position within a group or across the group boundary (see, e.g., Ng \& Maybery, 2002). Two types of errors are of particular interest in the assessment of the effect of grouping in sequences of six items: adjacent transpositions of an item within the first or last three items (1-2, 2-3, 4-5, and 5-6) and transpositions between Serial Positions 3 and 4 (3-4).

The next section deals with the effect of grouping on "1-2," "2-3," "4-5," and "5-6" errors on the one hand, and on " $3-4$ " errors on the other. The mean number of errors for each of these types was calculated across individual errors (across $1-2,2-3,4-5$, and 5-6 on the one hand, and across $3-4$ on the other) and analyzed using a 2 (condition) $\times 2$ (error type) repeated measures ANOVA. The main effect of condition was significant $[F(1,23)=$ $\left.6.02, M S_{\mathrm{e}}=0.86, p<.05\right]$, as was the interaction $\left[F(1,23)=9.04, M S_{\mathrm{e}}=0.89, p<.01\right]$, indicating that migrations $1-2,2-3,4-5$, and 5-6 were less frequent than migrations 3-4 in the ungrouped condition, whereas the reverse was the case in the grouped condition (see Figure 1, middle panel).

Response times. RTs for correctly recalled items were measured in milliseconds from the onset of the recall 

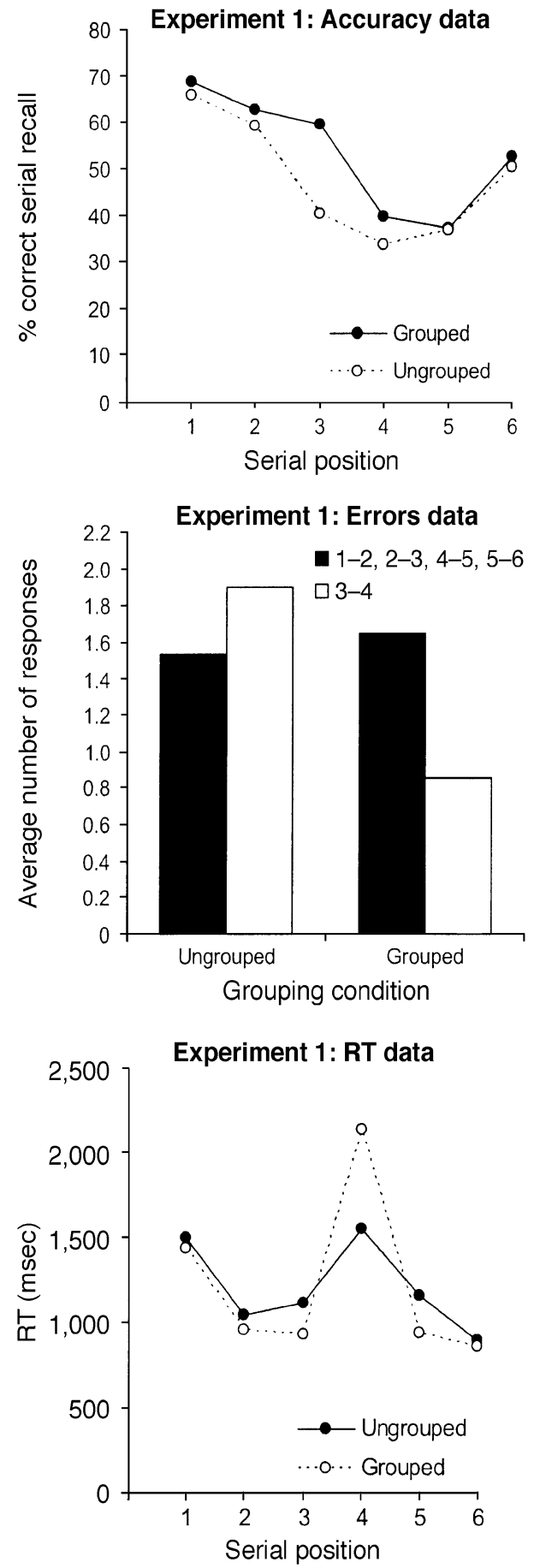

Figure 1. Experiment 1: recall accuracy (top panel), rates of "1-2," "2-3," "4-5," and "5-6" errors versus "3-4" errors (middle panel), and average median RTs for correctly recalled items (bottom panel) in the ungrouped and grouped conditions.

screen to the first response, and then from response to response. Median RTs were analyzed using a 2 (condition) $\times 6$ (serial position) repeated measures ANOVA. The effect of serial position was significant $[F(5,115)=$ $\left.9.88, M S_{\mathrm{e}}=659,889.4, p<.001\right]$, as was the interaction
$\left[F(5,115)=6.04, M S_{\mathrm{e}}=173,756.4, p<.001\right]$. As can be seen from Figure 1 (bottom panel), this interaction was due to the shorter RTs in the grouped condition in comparison with those in the ungrouped condition at all positions except the fourth, where the reverse effect was found. This was confirmed by the significant interaction of condition with the contrast of Serial Position 4 with Serial Positions 3 and $5\left[F(1,23)=6.91, M S_{\mathrm{e}}=717,211, p<.05\right]$.

\section{Discussion}

Serial recall of auditory spatial locations was enhanced by the combined temporal and pitch grouping manipulation, but primarily for the item immediately before the temporal gap separating the groups (i.e., at Serial Position 3). Migrations across the groups (3-4) were less frequent than other adjacent migrations in the grouped condition, whereas the opposite pattern of migrations was found in the ungrouped condition. A peak in RT was found in the grouped condition at the onset of the second group (note that a smaller peak was visible in the ungrouped condition, compatible with cross-condition contamination or spontaneous grouping).

In the absence of evidence from studies in which more extreme manipulations of timing were used, it could be argued that the peak in RT found at Serial Position 4 reflects (1) the reinstatement of encoding events, as is suggested by oscillator models (Brown et al., 2000; Burgess \& Hitch, 1999); (2) the time necessary to retrieve information about a list in order to organize its serial output (Anderson, Bothell, Lebiere, \& Matessa, 1998); or (3) the time to search mental sets before response output (Chuah \& Maybery, 1999; Cowan, 1992; Hulme, Newton, Cowan, Stuart, \& Brown, 1999), the two latter views being reinforced by recent evidence (Maybery et al., 2002).

In summary, in Experiment 1 we found solid evidence of the effect of grouping in four measures: accuracy (at Serial Position 3), shape of the serial position curve, errors, and RTs. Our strategy was to maximize the probability of detecting an effect by combining two grouping manipulations. Although an effect of grouping is demonstrated, the extent to which pitch and temporal grouping contribute to it remains open. In Experiment 2, four groups of participants were tested, each under one of four conditions: ungrouped, temporal grouping, pitch grouping, and combined temporal and pitch grouping. A betweenparticipants design eliminated potential contamination between the conditions.

\section{EXPERIMENT 2}

\section{Method}

Participants. Seventy-two 1st-year undergraduate psychology students at the University of Plymouth who reported normal hearing participated in exchange for course credit.

Apparatus and Materials. The experimental setup was similar to that used in Experiment 1. A set of 32 test sequences was built following the same rules as for that of Experiment 1.

Design. The ungrouped and combined conditions were as in Experiment 1 . The temporal and pitch grouping conditions were sim- 
ilar to the grouping condition of Experiment 1, with the differences that no pitch variation was used in the first and a regular rate of presentation (i.e., identical to that of the control condition) was applied to the second.

\section{Results}

Accuracy. The serial position curves (Figure 2, top panel) for the different conditions showed the U-shape usually found in order memory tasks. Only temporal grouping visibly improved accuracy of recall at Serial Position 3. Interestingly, the temporal and combined grouping conditions seemed to differ from the other conditions across Serial Positions 1-3 in terms of the shape of recall performance, indicating more pronounced bowing relative to performance in the ungrouped and pitch grouping conditions, for which accuracy appeared to decrease monotonically.

The percentage of correct responses was analyzed in a 4 (condition: ungrouped, temporal grouping, pitch grouping, combined grouping) $\times 6$ (serial position) ANOVA to test the observations described above. The effect of serial position was significant $\left[F(3,68)=126.71, M S_{\mathrm{e}}=\right.$ $69.54, p<.001]$, as was the interaction $[F(3,68)=3.82$, $\left.M S_{\mathrm{e}}=69.54, p<.001\right]$.

The effect of grouping condition at each serial position was analyzed, and a significant effect was found at Serial Position 3 only $\left[F(3,68)=5.77, M S_{\mathrm{e}}=116.25\right.$, $p<.005]$. Comparisons carried out at Serial Position 3 revealed significantly better performance in the temporal grouping condition in comparison with both the ungrouped condition $[F(1,68)=16.08, p<.001]$ and the pitch grouping condition $[F(1,68)=6.55, p<.05]$, and a significant advantage of the combined grouping condition over the ungrouped condition $[F(1,68)=6.55, p<.05]$.

\section{Classification of Errors}

The number of omissions, intrusions, and order errors, reported in the Appendix, confirmed the pattern of results observed in Experiment 1. With respect to the two types of order error predicted to be sensitive to grouping ("1-2," "2-3," "4-5," and "5-6" order errors vs. "3-4" order errors), grouping decreased the number of " $3-4$ " errors, an effect that was stronger in the temporal and combined grouping conditions than in the pitch grouping condition (Figure 2, middle panel). Grouping did not affect the number of within-group errors.

To confirm these observations, the mean number of errors was analyzed using a 4 (grouping condition) $\times 2$ (error type) ANOVA. The main effect of grouping condition was significant $\left[F(3,68)=6.07, M S_{\mathrm{e}}=1.20, p<\right.$ $.005]$, as was the main effect of error type $[F(1,68)=$ $\left.7.22, M S_{\mathrm{e}}=0.83, p<.01\right]$ and the interaction $[F(3,68)=$ $\left.7.23, M S_{\mathrm{e}}=0.83, p<.001\right]$. The analysis of the interaction revealed that all conditions led to similar numbers of " $1-2$," "2-3," "4-5," and "5-6" errors, whereas the effect of condition was significant for the "3-4" errors $\left[F(3,68)=9.25, M S_{\mathrm{e}}=1.40, p<.001\right]$. The number of "3-4" errors was significantly larger in the ungrouped condition than in the temporal grouping $[F(1,68)=22.94$,
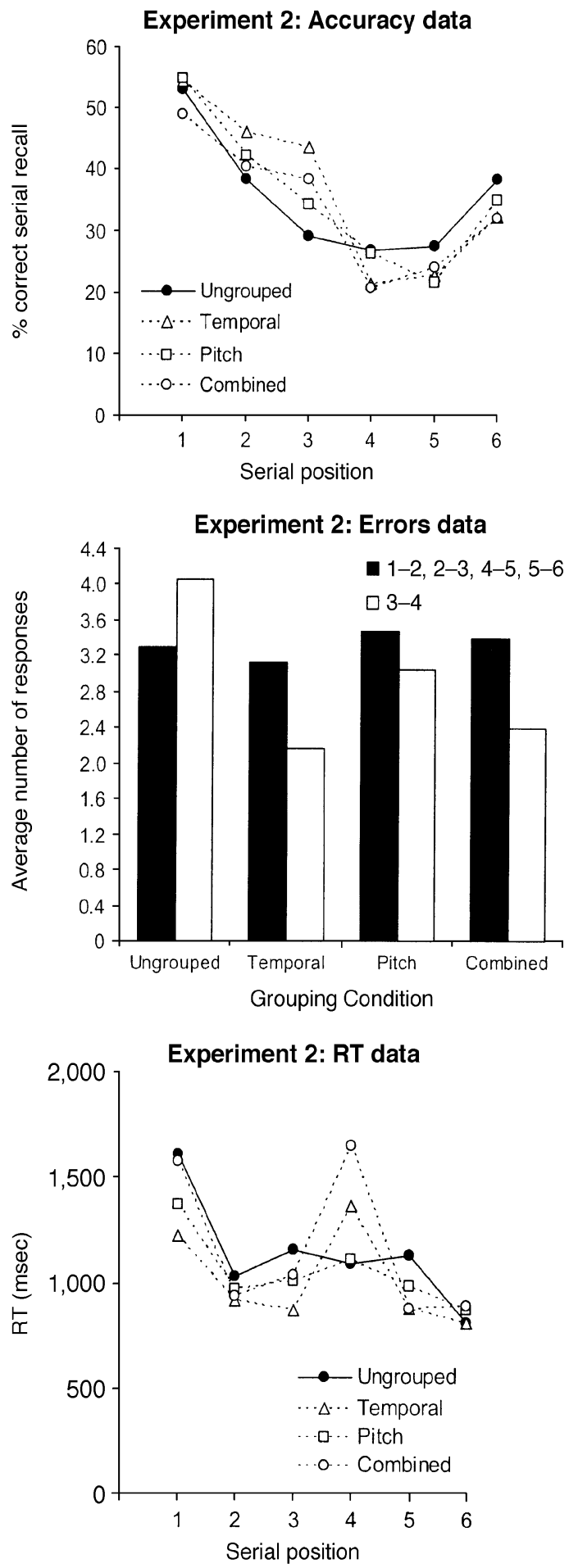

Figure 2. Experiment 2: recall accuracy (top panel), rates of "1-2," "2-3," "4-5," and "5-6" errors versus "3-4" errors (middle panel), and average median RTs for correctly recalled items (bottom panel) in the ungrouped, temporal grouping, pitch grouping, and combined grouping conditions.

$p<.001]$, pitch grouping $[F(1,68)=6.43, p<.05]$, and combined grouping $[F(1,68)=17.86, p<.001]$ conditions, and was larger in the pitch grouping than in the temporal grouping condition $[F(1,68)=5.0, p<.05]$. 
In summary, the probability of migrations between Serial Positions 3 and 4 was reduced in the pitch grouping condition relative to the ungrouped condition, and more so in the temporal grouping condition. The combined grouping condition produced a frequency of " $3-4$ " errors that did not differ from that observed in the temporal and pitch grouping conditions.

Response times. RTs revealed a remarkably clear pattern of results: A clear peak in RT was found at Serial Position 4 in the temporal and combined grouping conditions and a relatively modest peak appeared in the pitch condition, whereas no such peak emerged in the ungrouped condition.

A 4 (grouping condition) $\times 6$ (serial position) ANOVA carried out on the median correct RTs confirmed these observations. The effect of serial position was significant $\left[F(5,340)=40.54, M S_{\mathrm{e}}=94,524.5, p<.001\right]$, as was the interaction $\left[F(15,340)=3.99, M S_{\mathrm{e}}=94,524.5\right.$, $p<.001]$.

As in Experiment 1, a contrast between Serial Position 4 and Serial Positions 3 and 5 was considered in interaction with grouping condition to decompose the interaction. Differences were found between the temporal grouping and ungrouped conditions $[F(1,68)=8.83, p<.005]$, the temporal grouping and pitch grouping conditions $[F(1,68)=$ $4.29, p<.05]$, the combined grouping and ungrouped conditions $[F(1,68)=16.64, p<.001]$, and the combined and pitch grouping conditions $[F(1,68)=10.10, p<$ .005].

In summary, in the two conditions involving temporal grouping (temporal and combined grouping), the participants grouped their responses in two groups to similar degrees, whereas they did not do so in the pitch grouping and ungrouped conditions.

Discussion. The results of Experiment 2 suggest that temporal grouping was the key manipulation affecting recall accuracy (at Serial Position 3), errors, and RTs in Experiment 1. No evidence of the effect of pitch grouping was found on recall accuracy or RTs; only the probability of " $3-4$ " errors decreased in that condition in comparison with the ungrouped condition, but the decrease was not as pronounced as in the temporal or combined grouping condition. Finally, the temporal and combined grouping conditions did not differ significantly from each other on any of the decisive measures analyzed above, indicating that the combination of pitch and temporal grouping did not have an interactive effect. Finally, in Experiment 2 the use of spontaneous grouping in the ungrouped condition was successfully limited, as is suggested by the absence of a peak of RT at Serial Position 4.

\section{GENERAL DISCUSSION}

The results from Experiment 1 suggest that grouping affects performance in terms of accuracy (at Serial Position 3), the shape of the serial position curve, the nature of errors, and RTs in an auditory spatial order memory task. Experiment 2 established that this effect relates to temporal rather than pitch grouping.
Many aspects of our data suggest similarities between auditory verbal and spatial serial memory. The shape of the serial position curve in our ungrouped condition presents primacy and recency components, in line with the effects observed in other studies using nonverbal (e.g., Avons, 1998; Jones, Farrand, Stuart, \& Morris, 1995; Parmentier \& Jones, 2000) as well as verbal (e.g., Frankish, 1989) stimuli. More importantly, there are clear similarities between the effects of grouping in our experiments and those found in verbal studies. First, grouping enhances recall accuracy (see, e.g., Frankish, 1989), even though this effect was limited to the last item of the first group in our experiments. Second, the enhancement is selective inasmuch as the improved performance occurs particularly for the last item in a group, producing a characteristic scallop-shaped serial position curve (see, e.g., Hitch et al., 1996). Third, grouping reduces the probability of items migrating to adjacent positions if migration involves crossing from one subseries to another (see, e.g., Henson, 1998). Fourth, in line with some of our work on verbal serial memory, grouping of items at presentation translates into grouping in the timing of responses, resulting in a pause between groups (Maybery et al., 2002).

Functional similarities leave room for domain-specific characteristics, however. Our claim is not that verbal and spatial memories are identical, but that processes involving order memory (or temporal codes) and grouping overlap to a degree. Auditory verbal and spatial stimuli will inevitably differ in other characteristics. Our data seem to vary from the findings of verbal studies in two aspects of performance: the amplitude of the grouping effect is relatively smaller in our experiments, at least for accuracy, and the recency effect observed, though noticeable, is less pronounced than that found with auditory verbal lists. With regard to the first point, there may be factors enhancing overall accuracy specifically in grouped verbal lists, whereas other factors may benefit both verbal and spatial lists. For example, grouped verbal material can elicit the formation of chunks or higher order units (see, e.g., Cowan, 2001; McNicol \& Heathcote, 1986), whereas our stimuli, because they do not benefit from preexisting long-term memory representations, cannot capitalize on such a process. As for the recency effect, there are suggestions that modality differences in verbal memory could result from differential redintegration effects (Tehan \& Humphreys, 1995, 1998), making redintegration (the reconstruction of a degraded episodic trace through matching with long-term memory representations of items) a potential contributor to recency effects. ${ }^{1}$ By the same token, since no preexisting representations of our stimuli are available to participants, a redintegration process is hardly possible in our task.

Our results are compatible with the idea that grouping is the result of additional rehearsal time provided by the temporal separation between groups (see Lewandowsky $\&$ Brown, in press, for recent evidence indicating that the time available after an item, but not before, increases serial recall of that item), but they do not constitute irrefutable support for such a view. Auditory streaming, or 
the perceptual integration of auditory stimuli, offers an alternative account. Streaming applied to successive stimuli has important consequences for the encoding of order information. In particular, the order of events at the boundaries of auditory objects demarcated by some distinctive feature is particularly well encoded. The last item in that type of sequence seems to serve as an anchor for those immediately before it (Nicholls \& Jones, 2002). Our results might illustrate the effectiveness of our temporal manipulation in establishing boundaries in the auditory stream. This view suggests that boundary items benefit from some level of distinctiveness relative to their neighbors. The latter point also applies to the temporal distinctiveness theory (see, e.g., Glenberg \& Swanson, 1986; Neath \& Crowder, 1990), according to which the probability of recall of an item increases with its temporal distance from its neighbors. However, this theory, at least as it stands, predicts that the item following a temporal gap, not the one preceding it, would benefit from better recall (i.e., the opposite of what we have reported).

In summary, the present study establishes that temporal grouping affects serial memory for auditory-spatial stimuli and that pitch grouping apparently does not, or at least does so to a lesser extent. The effect of temporal grouping mimics that observed in verbal studies in four respects: the shape of the serial position function, some increase in recall accuracy, a specific pattern of errors, and the temporal grouping of responses.

\section{REFERENCES}

Anderson, J. R., Bothell, D., Lebiere, C., \& Matessa, M. (1998). An integrated theory of list memory. Journal of Memory \& Language, $\mathbf{3 8}, 341-380$.

Anderson, J. R., \& MATEssa, M. (1997). A production system theory of serial memory. Psychological Review, 104, 728-748.

Avons, S. E. (1998). Serial report and item recognition of novel visual patterns. British Journal of Psychology, 89, 285-308.

Brown, G. D. A., Preece, T., \& Hulme, C. (2000). Oscillator-based memory for serial order. Psychological Review, 107, 127-181.

Burgess, N., \& Hitch, G. (1999). Memory for serial order: A network model of the phonological loop and its timing. Psychological Review, 106, 551-581.

CHUAH, Y. M. L., \& MAYBERY, M. T. (1999). Verbal and spatial shortterm memory: Common sources of developmental change? Journal of Experimental Child Psychology, 73, 7-44.

Cowan, N. (1992). Verbal memory span and the timing of spoken recall. Journal of Memory \& Language, 31, 668-684.

Cowan, N. (2001). The magical number 4 in short-term memory: A reconsideration of mental storage capacity. Behavioral \& Brain Sciences, 24, 87-114.

FRANKISH, C. (1985). Modality-specific grouping effects in short-term memory. Journal of Memory \& Language, 24, 381-389.
FranKISH, C. (1989). Perceptual organization and PAS. Journal of Experimental Psychology: Learning, Memory, \& Cognition, 15, 469-479.

Glenberg, A. M., \& Swanson, N. G. (1986). A tempora distinctiveness theory of recency and modality effects. Journal of Experimental Psychology: Learning, Memory, \& Cognition, 12, 3-15.

Henson, R. N. A. (1998). Short-term memory for serial order: The start-end model. Cognitive Psychology, 36, 73-137.

Hitch, G. J., Burgess, N., Towse, J. N., \& CuLPIN, V. (1996). Temporal grouping effects in immediate recall: A working memory analysis. Quarterly Journal of Experimental Psychology, 49A, 116-139.

Hulme, C., Newton, O., Cowan, N., Stuart, G., \& Brown, G. D. A. (1999). Think before you speak: Pauses, memory search, and trace reintegration processes in verbal memory span. Journal of Experimental Psychology: Learning, Memory, \& Cognition, 25, 447-463.

Jones, D., Farrand, P., Stuart, G., \& Morris, N. (1995). Functional equivalence of verbal and spatial information in serial short-term memory. Journal of Experimental Psychology: Learning, Memory, \& Cognition, 21, 1008-1018.

LASHLEY, K. S. (1951). The problem of serial order in behavior. In L. A. Jeffress (Ed.), Cerebral mechanisms in behavior: The Hixon symposium (pp. 112-136). New York: Wiley.

LEWANDOWSKY, S., \& BRown, G. D. A. (in press). Serial recall and presentation schedule: A micro-analysis of local distinctiveness. Memory.

Maybery, M. T., Parmentier, F. B. R., \& Jones, D. M. (2002). Grouping of list items reflected in the timing of recall: Implications for models of serial verbal memory. Journal of Memory \& Language, 47, 360-385.

MCNicol, D., \& Heathсote, A. (1986). Representation of order information: An analysis of grouping effects in short-term memory. Journal of Experimental Psychology: General, 115, 76-95.

NEATH, I., \& Crowder, R. G. (1990). Schedules of presentation and temporal distinctiveness in human memory. Journal of Experimental Psychology: Learning, Memory, \& Cognition, 16, 316-327.

NG, H. L. H., \& MAYBERY, M. T. (2002). Grouping in short-term verbal memory: Is position coded temporally? Quarterly Journal of Experimental Psychology, 55A, 391-424.

Nicholls, A. P., \& Jones, D. M. (2002). Capturing the suffix: Cognitive streaming in immediate serial recall. Journal of Experimental Psychology: Learning, Memory, \& Cognition, 28, 12-28.

OLDFIEld, S. R., \& PARKER, S. P. A. (1984). Acuity of sound localisation: A topography of auditory space. I. Normal hearing conditions. Perception, 13, 581-600.

PaGe, M. P. A., \& NorRIS, D. (1998). The primacy model: A new model of immediate serial recall. Psychological Review, 105, 761-781.

Parmentier, F. B. R., \& Jones, D. M. (2000). Functional characteristics of auditory temporal-spatial short-term memory: Evidence from serial order errors. Journal of Experimental Psychology: Learning, Memory, \& Cognition, 26, 222-238.

TeHAN, G., \& HumphreYs, M. S. (1995). Transient phonemic codes and immunity to proactive interference. Memory \& Cognition, 23, 181191.

Tehan, G., \& Humphreys, M. S. (1998). Creating proactive interference in immediate recall: Building a DOG from a DART, a MOP, and a FIG. Memory \& Cognition, 26, 477-489.

\section{NOTE}

1. We thank reviewer Gerry Tehan for pointing out the relevance of redintegration in accounting for modality differences in serial position curves. 


\section{APPENDIX \\ Analysis of Omissions, Intrusions, and Order Errors in Experiments 1 and 2}

\section{Analysis of Errors in Experiment 1}

The main effect of grouping was not significant $\left[F(1,23)=1.73, M S_{\mathrm{e}}=81.75, p>\right.$ .20]. The effect of error type was significant $\left[F(2,46)=205.97, M S_{\mathrm{e}}=57.61, p<.001\right]$. No interaction was present $\left[F(2,46)=1.01, M S_{\mathrm{e}}=43.19, p>.37\right]$.

\section{Analysis of Errors in Experiment 2}

The main effect of grouping was not significant $\left[F(3,68)<1, M S_{\mathrm{e}}=18.68, p>\right.$.87]. The effect of error type was significant $\left[F(2,138)=522.52, M S_{\mathrm{e}}=37.44, p<.001\right]$. No interaction was present $\left[F(6,136)<1, M S_{\mathrm{e}}=37.44, p>.90\right]$.

Table A1

Mean Total Numbers of Errors (and Standard Deviations) for the Different Grouping Conditions of Experiments 1 and 2

\begin{tabular}{|c|c|c|c|c|c|c|}
\hline \multirow[b]{2}{*}{ Condition } & \multicolumn{2}{|c|}{ Omissions } & \multicolumn{2}{|c|}{ Intrusions } & \multicolumn{2}{|c|}{ Order Errors } \\
\hline & $M$ & $S D$ & $M$ & $S D$ & $M$ & $S D$ \\
\hline \multicolumn{7}{|l|}{ Experiment 1} \\
\hline Ungrouped & 3.21 & 5.49 & 13.85 & 3.26 & 35.72 & 10.72 \\
\hline Grouped & 2.08 & 4.06 & 13.19 & 4.60 & 31.55 & 11.72 \\
\hline \multicolumn{7}{|l|}{ Experiment 2} \\
\hline Ungrouped & 2.03 & 2.80 & 18.34 & 2.28 & 35.47 & 9.87 \\
\hline Temporal grouping & 2.17 & 2.83 & 18.46 & 2.27 & 36.63 & 9.82 \\
\hline Pitch grouping & 3.27 & 3.77 & 18.34 & 2.51 & 35.62 & 6.91 \\
\hline Combined grouping & 2.40 & 5.04 & 19.33 & 2.78 & 34.00 & 5.68 \\
\hline
\end{tabular}

(Manuscript received July 5, 2001;

revision accepted for publication June 11, 2003.) 